\title{
Feasibility of esophageal stent fixation with an over-the- scope-clip for malignant esophageal strictures to prevent migration
}

\section{(ㄷ)(1) $(9)$}

\author{
Authors \\ Hiromasa Ohira² \\ Institutions \\ 1 Department of Endoscopy, Fukushima Medical \\ University Hospital, Fukushima, Japan \\ 2 Department of Gastroenterology, Fukushima Medical \\ University School of Medicine, Fukushima, Japan \\ 3 Department of Advanced Gastrointestinal Endoscopy, \\ Fukushima Medical University, Fukushima, Japan
}

Ko Watanabe ${ }^{1,2}$, Takuto Hikichi ${ }^{1}$, Jun Nakamura1,2, Tadayuki Takagii ${ }^{2}$, Rei Suzuki ${ }^{2}$, Mitsuru Sugimoto ${ }^{2}$, Yuichi

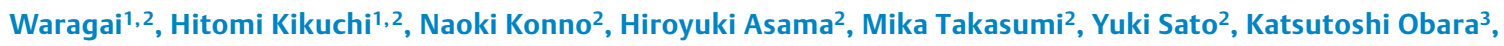

submitted 3.1.2017

accepted after revision 9.5.2017

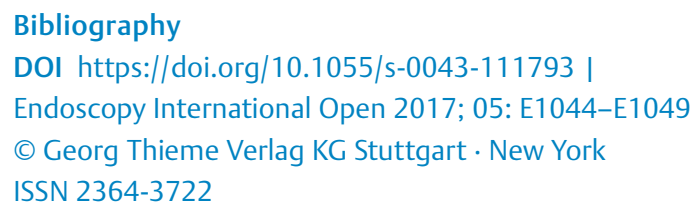

Corresponding author

Takuto Hikichi, MD, PhD, Director and Associate Professor, Department of Endoscopy, Fukushima Medical University Hospital, 1 Hikarigaoka, Fukushima, Japan, 960-1295

Fax: +81-24-547-1586

takuto@fmu.ac.jp

\section{ABSTRACT}

Background and study aims Fixation of an esophageal self-expandable metal stent (SEMS) with an over-the- scope-clip (OTSC) system for a benign stricture to prevent migration has been reported. However, the efficacy of SEMS fixation with an OTSC for malignant esophageal stricture remains unclear. The aim of this retrospective study was to evaluate the feasibility of SEMS fixation with an OTSC for a malignant esophageal stricture.

Patients and methods Twelve patients who underwent esophageal SEMS placement and fixation with an OTSC for a malignant esophageal stricture were included in this retrospective study. The primary endpoint was technical success. The secondary endpoint was clinical success, which was defined as an improvement of at least 1 grade in the dysphagia score 1 week after SEMS placement or changes in the dysphagia score from before SEMS placement to 1 week after SEMS placement.

Results The technical success rate was $100 \%$. The clinical success rate was $92.3 \%$. In 6 mild stricture cases in which a standard peroral endoscope could be used, no migration of the SEMS was observed. The median dysphagia score before and at 1 week after SEMS placement was 3 (range $2-4$ ) and $0(0-4)$, respectively, which indicated improvement at 1 week after SEMS placement compared with before SEMS placement $(P=0.002)$. There were no adverse events associated with placement of SEMS and deployment of an OTSC. Conclusions SEMS fixation with an OTSC is feasible for prevention of migration due to a malignant esophageal stricture.

\section{Introduction}

Dysphagia is a major symptom in patients with a malignant esophageal stricture, such as esophageal carcinoma, gastroesophageal junction carcinoma, gastric cardia carcinoma, and esophageal metastasis from other carcinomas, and it causes poor nutrition intake and a decrease in quality of life. Esophageal self-expandable metal stent (SEMS) placement has been widely used for palliative treatment of unresectable malignant esophageal strictures to relieve dysphagia and increase nutritional intake [1 - 3]. Recently, a clinical guideline for esophageal stents was published by the European Society of Gastrointestinal Endoscopy (ESGE) [4]. In this guideline, ESGE recommended placement of a partially or fully covered SEMS for palliative treatment of malignant dysphagia over laser therapy, photodynamic therapy, and esophageal bypass. However, migration is one of the most common adverse events (AE) after SEMS placement, and its prevalence ranges from $4-36 \%$ [2, 3, 5-7]. Several researchers have reported fully covered stent placement, concurrent chemotherapy and/or radiotherapy and stents placed across the gastroesophageal junction as factors that increase risk of stent migration [5, 6, 8, 9]. Additionally, the gen- 
eral indication of esophageal SEMS placement for unresectable malignant esophageal stricture is severe dysphagia in cases in which the stricture cannot allow the passage of a standard peroral endoscope to prevent migration [3,8]. However, in clinical practice, we have often encountered malignant esophageal strictures in patients with severe dysphagia; however, the stricture is often mild and can allow the passage of a standard peroral endoscope. In such mild stenosis cases with severe dysplasia, we face the problem regarding the suitable timing of stent placement because of the risk of migration.

Recently, a new endoscopic clipping device called the overthe-scope-clip (OTSC) system (Ovesco Endoscopy, Tübingen, Germany) has become available for the closure of perforations, anastomotic leaks, and fistulas $[10,11]$. The OTSC system has a stronger closing force than the through-the-scope hemostatic clip [12]. Therefore, some researchers have reported esophageal SEMS fixation with an OTSC to prevent migration [1013]. However, in these reports, the main indications for stenting were postoperative leak, fistula, perforation and benign strictures. Few reports have discussed efficacy or safety of esophageal SEMS fixation with an OTSC for malignant esophageal strictures.

Therefore, we performed a study on the feasibility of esophageal SEMS fixation with an OTSC for malignant esophageal strictures.

\section{Patients and methods}

\section{Patients}

Twelve consecutive patients underwent esophageal SEMS placement and fixation with an OTSC for malignant esophageal strictures and 13 procedures were performed at Fukushima Medical University Hospital between September 2014 and October 2016. Procedural and clinical data were collected and analyzed retrospectively from a prospectively maintained endoscopy database. The inclusion criteria for patients for SEMS placement were as follows: 1) dysphagia and a dysphagia score of at least 2 (dysphagia scores: 0 , no dysphagia; 1, dysphagia to normal solid food; 2 , dysphagia to soft solid foods; 3 , dysphagia also with liquids; and 4, aphagia, inability to swallow saliva) [14]; 2) presence of a malignant esophageal stricture that did not allow passage of the endoscope, which had a diameter of 9.2 mm (GIF-Q260; Olympus Medical Systems Corp., Tokyo, Japan), or could barely allow the passage of the scope; 3 ) ineligibility for curative surgery because of advanced or metastatic disease or poor functional status; and 4) lack of exposure to initial chemotherapy and/or radiotherapy. The exclusion criteria were as follows: 1) a stricture within $2 \mathrm{~cm}$ from the upper esophageal sphincter; 2 ) radiotherapy or chemotherapy within 1 month prior to SEMS placement; and 3) refusal to undergo SEMS placement and fixation with an OTSC. SEMS fixation with an OTSC was performed in all SEMS placement patients who consented to use the OTSC in this study period. All patients provided written informed consent before the procedure, and this study was conducted with the approval of the Ethics Committee of Fukushima Medical University (approval No. 2496).
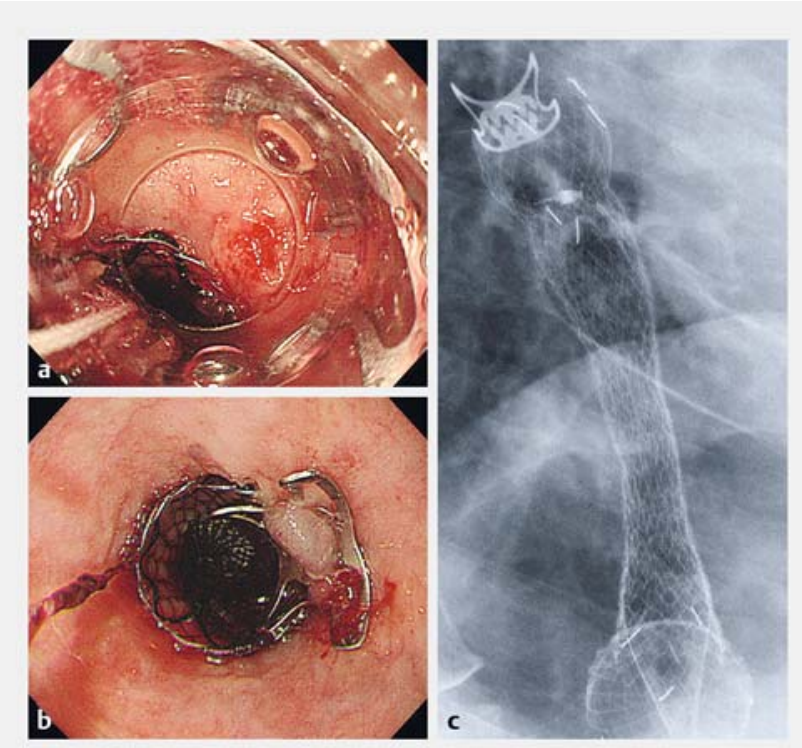

- Fig. 1 a An endoscopic image. The over-the-scope-clip (OTSC) system was loaded onto the scope, and part of the upper rim of the stent was suctioned into the transparent cap before releasing the OTSC. b An endoscopic image. The OTSC was released, grasping both the self-expandable metal stent and esophageal wall. c An $\mathrm{X}$-ray image after the OTSC and stent placement.

\section{SEMS placement and fixation with an OTSC}

All procedures were performed with patients under deep sedation using midazolam. The lesion was inspected with an endoscope (GIF-Q260, GIF-Q260 J, GIF-2T240; Olympus Medical Systems Corp., Tokyo, Japan) to determine the location and length of the stenosis. Marking clips were placed with endoscopy at the upper end of the stricture. In mild stricture cases that allowed the passage of the endoscope, marking clips were also placed in the lower end. A stent that was at least $4 \mathrm{~cm}$ longer than the stricture was used to allow at least a $2-\mathrm{cm}$ extension above and below the proximal and distal tumor margins. The stent was positioned over a guidewire and deployed under fluoroscopy guidance and, in some cases, also under endoscopy guidance. Subsequently, the OTSC system was loaded onto the scope and part of the upper rim of the stent was suctioned into the transparent cap before releasing the OTSC, grasping both the SEMS and esophageal wall ( $\triangleright$ Fig. 1). We avoided deploying the OTSC in areas of pulsations to prevent potential grasping of the vasculature structure, as noted in a previous report [12]. Only a single OTSC was placed per patient. In all cases, the Niti-S stent (Taewoong Medical, Seoul, Korea) was used and the type of stents were as follows: a long, covered stent $(n=10)$, partially covered stent $(n=2)$, and fully covered stent $(n=1)$, with diameters of $18 \mathrm{~mm}$ and lengths of 80 to $150 \mathrm{~mm}$. The type of OTSC was $11 / 6 \mathrm{t}$, which had an OTSC cap diameter of $16.5 \mathrm{~mm}$ and clip width of $9 \mathrm{~mm}$, and the type of teeth was ' $t$ ' in all cases. 
- Table 1 Patient characteristics.

\begin{tabular}{|c|c|c|c|c|c|c|c|}
\hline Case & Age & Gender & $\begin{array}{l}\text { Source of malignant } \\
\text { esophageal stricture }\end{array}$ & Location & $\begin{array}{l}\text { Passage of } \\
\text { GIF-Q260 }\end{array}$ & $\begin{array}{l}\text { DS before } \\
\text { SEMS } \\
\text { placement }\end{array}$ & Pretreatment \\
\hline 1 & 70 & Male & Esophageal squamous cell carcinoma & Middle & Impossible & 4 & Chemoradiotherapy \\
\hline 2 & 79 & Male & Esophageal adenocarcinoma & Lower & Possible & 3 & Chemotherapy \\
\hline 3 & 57 & Male & Esophageal endocrine cell carcinoma & Lower & Impossible & 3 & Chemotherapy \\
\hline 4 & 52 & Male & Lymph node metastasis of lung carcinoma & Middle & Impossible & 3 & Chemoradiotherapy \\
\hline 5 & 77 & Female & Esophageal adenosquamous cell carcinoma & Lower & Possible & 4 & None \\
\hline 6 & 53 & Female & Gastroesophageal junction carcinoma & Lower & Possible & 4 & Chemotherapy \\
\hline 7 & 48 & Male & Esophageal adenocarcinoma & Lower & Impossible & 2 & Chemotherapy \\
\hline 8 & 83 & Male & Esophageal squamous cell carcinoma & Lower & Impossible & 2 & None \\
\hline 9 & 75 & Male & Esophageal squamous cell carcinoma & Middle & Possible & 2 & Chemoradiotherapy \\
\hline 10 & 84 & Male & Esophageal adenocarcinoma & Lower & Impossible & 3 & None \\
\hline 11 & 67 & Male & Esophageal squamous cell carcinoma & Lower & Possible & 2 & Chemoradiotherapy \\
\hline 12 & 74 & Male & Esophageal squamous cell carcinoma & Lower & Possible & 2 & Chemoradiotherapy \\
\hline
\end{tabular}

\section{Outcomes}

The primary endpoint was technical success. The secondary endpoints were clinical success, changes in the dysphagia score from before SEMS placement to 1 week after SEMS placement, and adverse events (AE), including migration. Technical success was defined as adequate deployment and positioning of the SEMS at the site of the stricture and ability to deploy the OTSC and fix the SEMS. Clinical success was defined as an improvement of at least 1 grade in the dysphagia score 1 week after SEMS placement. Stent migration was defined as movement out of the stricture, which was diagnosed on endoscopy and radiography. Follow-up information after SEMS placement was collected from medical records. If patients were followed up outside of our institution, we conducted a questionnaire survey with their primary care physicians. The procedure time required for OTSC placement was calculated from endoscope insertion after SEMS deployment to final endoscope withdrawal after OTSC placement.

\section{Statistical analysis}

Values are reported as the medians with ranges. Changes in dysphagia scores from before SEMS placement to 1 week after SEMS placement were analyzed using the Wilcoxon's signedrank test. Differences were considered to be significant at $P<0.05$. This analysis was performed using the SPSS software (version 21 for windows; IBM Corp, Armonk, NY, USA).

\section{Results}

Patient characteristics are summarized in $>$ Table1. The median age of the patients ( 10 men, 2 women) was 70 years (range $48-83$ years). The source of malignant esophageal stricture included esophageal carcinoma in 10 patients ( 5 squamous cell carcinoma, 3 adenocarcinoma, 1 adenosquamous cell carcinoma, and 1 endocrine cell carcinoma), gastroesophageal junction carcinoma in one patient, and lymph node metastasis of lung carcinoma in 1 patient. Before SEMS placement, 5 patients had received chemoradiotherapy, 4 patients had received only chemotherapy, and 3 patients had received only the best supportive care. The median period from last chemotherapy to SEMS placement in chemoradiotherapy or chemotherapy cases was 1 month (range 1-23 months). The main locations of the strictures were the lower esophagus in 9 patients and middle esophagus in 3 patients. In 6 patients (7 SEMS placements), the stricture allowed passage of the endoscope, which had a diameter of $9.2 \mathrm{~mm}$ (GIF-Q260; Olympus Medical Systems Corp., Tokyo, Japan) before SEMS placement.

Treatment outcomes and AEs are summarized in > Table 2 . The technical success rate was $100 \%$ (12/12), and successful application of the OTSC was accomplished in all patients and all SEMS placements. Median procedure time required for OTSC placement was 11 minutes (range 6-15 minutes). The clinical success rate was $92.3 \%(11 / 12)$, and 11 patients showed an improved dysphagia score after SEMS placement. Only 1 patient did not improve clinically. Median dysphagia score before and at 1 week after SEMS placement was 3 (range $2-4)$ and $0(0-4)$, respectively, which indicated improvement at 1 week after SEMS placement compared with before SEMS placement $(P=0.002)$. Furthermore, in mild stricture cases 
- Table 2 Treatment outcomes and adverse events.

\begin{tabular}{|c|c|c|c|c|c|c|c|c|}
\hline Case & $\begin{array}{l}\text { Techni- } \\
\text { cal suc- } \\
\text { cess }\end{array}$ & $\begin{array}{l}\text { Clinical } \\
\text { success }\end{array}$ & $\begin{array}{l}\text { Procedure time } \\
\text { for OTSC place- } \\
\text { ment, min }\end{array}$ & Type of SEMS & $\begin{array}{l}\text { Length } \\
\text { of SEMS, } \\
\text { mm }\end{array}$ & $\begin{array}{l}\text { DS after SEMS } \\
\text { placement }\end{array}$ & $\begin{array}{l}\text { Adverse } \\
\text { events }\end{array}$ & $\begin{array}{l}\text { Concurrent } \\
\text { chemother- } \\
\text { apy }\end{array}$ \\
\hline 1 & Yes & Yes & 11 & Partially covered & 80 & 0 & None & No \\
\hline 2 & Yes & Yes & 12 & Long covered & 120 & 0 & None & No \\
\hline $3-1$ & Yes & Yes & 10 & Long covered & 100 & 0 & None & Yes \\
\hline $3-2$ & Yes & Yes & 15 & Long covered & 100 & 0 & None & No \\
\hline 4 & Yes & Yes & 9 & Partially covered & 100 & 1 & None & No \\
\hline 5 & Yes & No & 11 & Long covered & 150 & 4 & None & No \\
\hline 6 & Yes & Yes & 8 & Long covered & 80 & 0 & None & Yes \\
\hline 7 & Yes & Yes & 12 & Long covered & 100 & 1 & None & No \\
\hline 8 & Yes & Yes & 13 & Long covered & 120 & 0 & None & No \\
\hline 9 & Yes & Yes & 13 & Fully covered & 80 & 0 & None & Yes \\
\hline 10 & Yes & Yes & 9 & Long covered & 80 & 0 & None & No \\
\hline 11 & Yes & Yes & 8 & Long covered & 150 & 0 & None & No \\
\hline 12 & Yes & Yes & 6 & Long covered & 120 & 0 & None & No \\
\hline
\end{tabular}

that allowed passage of a GIF Q260 endoscope, median dysphagia score before and at 1 week after SEMS placement was 3 (range $2-4$ ) and $0(0-4)$, respectively, which also indicated improvement at 1 week after SEMS placement compared with before SEMS placement $(P=0.026)$.

There were no AEs associated with the placement of the SEMS and the deployment of the OTSC. After SEMS placement and OTSC deployment, 4 patients required analgesic agents, such as acetaminophen, for mild chest pain. All 4 patients were able to tolerate pain after medication and did not require any analgesic agents during the following days. The median clinical follow-up period after SEMS placement was 2 months (range 1-12). Three patients underwent chemotherapy and 9 patients received the best supportive care. Nine patients died of their underlying carcinoma. Recurrence of dysphagia occurred in 1 patient. In this case, SEMS placement with an OTSC was performed twice. The initial OTSC and SEMS were removed 12 months after the first SEMS placement because of obstruction by a tumor, and another SEMS was placed with an OTSC. OTSC removal was safely performed using a snare and gripping forceps. No delayed AEs, including migration of the SEMS, were observed during the follow-up period.

\section{Discussion}

This study showed that SEMS placement and fixation with an OTSC for malignant esophageal strictures was successful in all cases and that $92.3 \%$ of the patients had an improved dysphagia score. There were no AEs. In particular, there were no SEMS migrations. Nevertheless, a SEMS placed across the gastroesophageal junction because of stricture of the lower esopha- gus, mild stricture cases, a fully covered stent, and patients who underwent chemotherapy after SEMS placement, which were reported as the risk factors for SEMS migration, were included $[5,6,8,9]$.

According to the ESGE guideline [4], SEMS placement has not been recommended as a bridge to surgery, during concurrent use of radiotherapy, or prior to preoperative chemoradiotherapy, which has been associated with a high incidence of AEs. Our indications agreed with this guideline. The ESGE guideline described the controversy over whether SEMS placement after chemoradiotherapy is associated with the risk of major AEs; however, some studies have shown an increased risk of AEs. Therefore, we specifically informed patients who underwent chemoradiotherapy of the fatal AE risk of SEMS placement before the procedure.

Some techniques for SEMS fixation have been reported to prevent migration. Shim et al. [15] reported a technique consisting of a modified stent that was designed with a silk thread attached to the edge of the proximal end of the stent. Because this stent may not be routinely available, modifications of this technique using dental floss have also been reported [16]. With these techniques, after SEMS placement, the silk thread or dental floss is fixed to the patient's earlobe or nose, and the external fixation is removed at least 2 weeks after the procedure. Several researchers have reported the efficacy of SEMS fixation with through-the-scope hemostatic clips $[17,18]$. They have shown that rates of SEMS migration range from $0 \%-13 \%$. However, the authors fixed the SEMS by using 2 to 4 hemoclips because of the limited opening widths, closure strengths, and depths of penetration [12]. Recently, new SEMS fixation techniques using an OTSC, which is a new endoscopic 
clipping device that has a strong force, have been reported [10-13]. The main indications for stenting in these reports were benign diseases, such as postoperative leak, fistula, perforation and benign strictures. In these reports, OTSC application was successful in all patients. Irani et al. [12] and Mudumbi et al. [13] reported that SEMS migration occurred in $15.4 \%$ and $16.7 \%$ of patients, respectively, in SEMS fixed with an OTSC for benign esophageal strictures.

In our study, successful application of the OTSC was accomplished in all cases, as found in previous reports. SEMS fixed with an OTSC was easily and rapidly accomplished because an OTSC is easily placed by attaching it to the upper rim of the SEMS and esophageal wall as an endoscopic variceal ligation, and only a single OTSC was placed per patient. SEMS fixed with an OTSC was performed safely in all cases, and there were no SEMS migrations of the malignant esophageal strictures. Of the 6 mild stricture cases (7 SEMS placements) whose stricture allowed the passage of the endoscope, which had a diameter of $9.2 \mathrm{~mm}, 5$ cases (6 SEMS placements) showed improved dysphagia scores, indicating their improved quality of life. Only 1 case of esophageal adenosquamous carcinoma, which extensively invaded the stomach, did not improve clinically. However, even with a mild stricture, if a patient suffers from severe dysphagia, SEMS placement for a malignant esophageal stricture and fixation with an OTSC to prevent migration may help improve quality of life. Additionally, in mild stricture cases, it is possible to place marking clips in the lower end of the stricture, which may be useful for determining a suitable position for a SEMS.

Recently, Reijm et al. [19] reported that SEMS for malignant esophageal stricture results in moderate to severe pain, which is probably related to the instant expansion of SEMS, in $60 \%$ of patients after SEMS placement. In this study, chest pain requiring analgesic agents after the procedure was observed in 4 patients (33.3\%); however, no severe pain was observed. We considered the pain to be related to expansion of the SEMS, not grasping the esophageal wall with the OTSC, because the pain gradually decreased over time, similar to a previous report [19].

In the 1 case of recurrence of dysphagia, OTSC removal was safely performed using a snare and gripping forceps. Several researchers have also described a method for OTSC removal by cutting the hinge using argon plasm coagulation at 90 to $100 \mathrm{~W}$ $[12,20]$. Another researcher also described a method that uses an injector needle to create a submucosal cushion below the OTSC followed by use of a needle knife or exposed tip of a snare to make an incision below the OTSC into the submucosal cushion [13].

In this study, a fully covered stent was used in only 1 middle esophageal stricture case. Long covered stents were used in 10 procedures across the gastroesophageal junction to prevent gastroesophageal reflux. Partially covered stents were used in 2 cases of middle esophageal stricture to sufficiently grasp the upper rim of the stent using the OTSC because the upper rim of the partially covered stent was bare and was an uncovered region. In terms of the effect of fixation with an OTSC with a fully covered stent that does not have an uncovered lesion, additional studies are necessary.
This study has some limitations. First, it was small, had a retrospective design and was conducted at a single institution. Second, we did not compare the outcomes between fixation in the OTSC group and non-fixation group. Third, it remains unclear whether OTSC removal is both possible and safe at any time because reports on OTSC removal comprise small sample sizes $[12,13,20]$. Further prospective studies are necessary to confirm the effect of fixation with an OTSC. Finally, OTSC costs 79,800 Japanese yen (=approximately 720 US dollars), which makes it very expensive.

\section{Conclusions}

In conclusion, SEMS fixation with an OTSC to prevent migration for malignant esophageal strictures is feasible, safe, and easy. Further additional studies are necessary to confirm the effect of fixation with an OTSC.

\section{Acknowledgements}

The authors thank all of the endoscopy medical staff at Fukushima Medical University Hospital for their collaboration and assistance with the endoscopic procedures.

\section{Competing interests}

None

References

[1] Evans JA, Early DS, Chandraskhara V et al. The role of endoscopy in the assessment and treatment of esophageal cancer. Gastrointest Endosc 2013; 77: $328-334$

[2] Hindy P, Hong J, Lam-Tsai Y et al. A comprehensive review of esophageal stents. Gastroenterol Hepatol 2012; 8: 526-534

[3] Na HK, Song HY, Kim JH et al. How to design the optimal self-expandable oesophageal metallic stents: 22 years of experience in 645 patients with malignant strictures. Eur Radiol 2013; 23: 786-796

[4] Spaander MC, Baron TH, Siersema PD et al. Esophageal stenting for benign and malignant disease: European Society of Gastrointestinal Endoscopy (ESGE) Clinical Guideline. Endoscopy 2016; 48: 939-948

[5] Martins BC, Retes FA, Medrado BF et al. Endoscopic management and prevention of migrated esophageal stents. World J Gastrointest Endosc 2014; 6: 49-54

[6] Vleggaar FP, Siersema PD. Expandable stents for malignant esophageal disease. Gastrointest Endosc Clin N Am 2011; 21: 377-388

[7] Schoppmann SF, Langer FB, Prager $G$ et al. Outcome and complications of long-term self-expanding esophageal stenting. Dis Esophagus 2013; 26: $154-158$

[8] Park JH, Song HY, Kim JH et al. Polytetrafluoroethylene-covered retrievable expandable nitinol stents for malignant esophageal obstructions: factors influencing the outcome of 270 patients. AJR Am J Roentgenol 2012; 199: 1380 - 1386

[9] Spinelli P, Cerrai FG, Ciuffi M et al. Endoscopic stent placement for cancer of the lower esophagus and gastric cardia. Gastrointest Endosc 1994; 40: $455-457$ 
[10] Monkemuller K, Peter S, Toshniwal ] et al. Multipurpose use of the 'Bear claw' (over-the-scope-clip system) to treat endoluminal gastrointestinal disorders. Dig Endosc 2014; 26: 350 - 357

[11] Sulz MC, Bertolini R, Frei R et al. Multipurpose use of the over-thescope-clip system ("Bear claw") in the gastrointestinal tract: Swiss experience in a tertiary center. World J Gastroenterol 2014; 20: $16287-16292$

[12] Irani S, Baron TH, Gluck $M$ et al. Preventing migration of fully covered esophageal stents with an over-the-scope clip device (with videos). Gastrointest Endosc 2014; 79: 844-851

[13] Mudumbi S, Velazquez-Avina J, Neumann $\mathrm{H}$ et al. Anchoring of selfexpanding metal stents using the over-the-scope clip, and a technique for subsequent removal. Endoscopy 2014; 46: 1106 - 1109

[14] Knyrim K, Wagner HJ, Bethge $\mathrm{N}$ et al. A controlled trial of an expansile metal stent for palliation of esophageal obstruction due to inoperable cancer. N Engl J Med 1993; 329: $1302-1307$

[15] Shim CS, Cho YD, Moon JH et al. Fixation of a modified covered esophageal stent: its clinical usefulness for preventing stent migration. Endoscopy 2001; 33: 843-848
[16] da Costa Martins B, Medrado BF, de Lima MS et al. Esophageal metallic stent fixation with dental floss: a simple method to prevent migration. Endoscopy 2013; 45: E342

[17] Kato H, Fukuchi M, Miyazaki T et al. Endoscopic clips prevent self-expandable metallic stent migration. Hepato-Gastroenterology 2007; 54: $1388-1390$

[18] Vanbiervliet G, Filippi J, Karimdjee BS et al. The role of clips in preventing migration of fully covered metallic esophageal stents: a pilot comparative study. Surg Endosc 2012; 26: 53-59

[19] Reijm AN, Didden P, Bruno MJ et al. Early pain detection and management after esophageal metal stent placement in incurable cancer patients: A prospective observational cohort study. Endosc Int Open 2016; 4: E890 - 894

[20] Baron TH, Song LM, Ross A et al. Use of an over-the-scope clipping device: multicenter retrospective results of the first U.S. experience (with videos). Gastrointest Endosc 2012; 76: 202-208 